\begin{tabular}{|c|c|c|}
\hline & ORIGINAL RESEARCH PAPER & Surgery \\
\hline & $\begin{array}{l}\text { ROLE OF HYPERBILIRUBINEIMIA, ELEVATED C- } \\
\text { REACTIVE PROTEIN AND LEUCOCYTE COUNT } \\
\text { AS A PREDICTIVE FACTOR FOR APPENDICEAL } \\
\text { PERFORATION IN ACUTE APPENDICITIS }\end{array}$ & $\begin{array}{l}\text { KEY WORDS: Appendicitis, } \\
\text { Bilirubin, CRP, Perforation. }\end{array}$ \\
\hline
\end{tabular}

Nagendra Singh Bhadauria

Pawan Kumar

\section{Gunjan Sharma}

Amit Singh*
Assistant Professor, Department of Surgery, J.L.N Medical College, Ajmer, Rajasthan,India, 305001

Postgraduate resident, Department of Surgery, J.L.N Medical College, Ajmer, Rajasthan,India, 305001

Senior Resident Academic (Mch), Department of Paediatric Surgery, S.M.S Medical College Jaipur, Rajasthan. 302015.

Associate Professor, Department of Surgery, R.V.R.S Government Medical College, Bhilwara, Rajasthan, India 311001 * Corresponding Author

\begin{abstract}
Background: Appendiceal perforation in patients with acute appendicitis may cause a variety of potentially lifethreatening complications. Escherichia coli endotoxin has been shown to impact physiological bile flow in vivo. This had led to the theory that hyperbilirubinemia and elevated CRP in patients with appendicitis may have a predictive potential for the preoperative diagnosis of appendiceal perforation. Objectives: The aim of this retrospective study was to investigate the diagnostic value of hyperbilirubinemia and elevated CRP as a preoperative laboratory marker for appendiceal perforation in patients with acute appendicitis. Thus a new marker for appendiceal perforation would be welcomed. Thus, the need for the study is to conclude whether the Serum Bilirubin or CRP can be considered as a new laboratory marker to aid in the diagnosis of Appendicular Perforation. Materials and methods: All patients admitted with clinical diagnosis of "Acute Appendicitis" or "Appendicular Perforation" and undergoing surgery in General Surgery Dept in Tertiary Care Teaching Hospital were taken as Subjects for this study. The primary data for this study were the blood investigations of the patients. 200 patients with concerned consent and meeting the inclusion criteria were included in the study. The necessary clinical and lab details were obtained in a prewritten format. The intraoperative findings were noted and documented precisely. Upon completion of data collection the preoperative bilirubin levels of the perforated and uncomplicated groups wereOther clinical and lab parameters obtained during the study were compared between the two groups. All parameters were subjected to statistical analysis and evaluated critically. Results: A total of 200 patients participated in the study. Of the 200 patients studied 132 patients were males and 68 patients were females. The youngest patient was 13 years old and the oldest 64 years. Among all the patients operated with a diagnosis of Acute Appendicitis 92 were found to have a perforated appendix, and 108 were found to have a non-perforated appendix. It was observed that among 92 patients with perforated appendix 86 patients showed a raised CRP levels and 58 patients had a raised Bilirubin levels. However in case of non-perforated cases it was found that among the 108 cases 82 patients had a raised CRP levels and 36 patients showed raised bilirubin levels. Conclusion: Hence Logistic Regression technique revealed that the C- reactive protein and Hyperbilirubinemia can be used to predict the appendicular perforation preoperatively and that their roles are comparable and that there is no definitive advantage of one over the other for the same.
\end{abstract}

\section{Introduction}

The Vermiform Appendix is considered by most to be a vestigial organ. Its importance in surgery is due only to its propensity for inflammation, which results in the clinical syndrome known as Acute Appendicitis. Acute appendicitis is the most common cause of acute surgical abdomen leading to emergency laparotomy. Acute appendicitis presents as acute abdominal pain in the emergency care unit, the surgeon must realize that the reliance is based entirely on the clinical features rather than the lab or radiological findings. It is a very good aphorism that, in the diagnosis of acute abdominal conditions special investigation can be used only to reinforce the clinical diagnosis, seldom if ever can they establish or refute it. This is particularly appropriate in case of acute appendicitis. Diagnostic difficulties may lead to negative appendicectomies [1] or cases of missed appendi-citis resulting in complications such as appendiceal perforation (AP) or abscess formation [2].

Classic story of acute appendicitis is the onset of colicky abdominal pain followed by nausea or one or more episodes of vomiting with pain after several hours shifting to the lower right abdomen. Exactly the same clinical features are seen in other causes lower right sided inflammatory disorders.

The diagnostic and discriminatory role of white cell count (WCC) and C-reactive protein (CRP) in AA has been studied expansively but still remains contentious [3-7]. Literature points that a rise in serum bilirubin level in patients with clinically suspected appendicitis may be a predictor for perforation of appendix [8-13].

So many factors are found to affect the natural history of acute appendicitis like etiological factors, complication associated bacterial flora, antibiotic need etc. the greatest difficulty lies with in young children, elderly persons, and pregnant women. It should be stressed of course that physical signs of acute appendicitis is not specific but are those produced by local peritoneal irritation in the right iliac fossa the common cause of which is acute inflammation of the appendix. So, it is imperative that a rationalized model to aid the clinical decision making is devised in an age accustomed to early and accurate preoperative diagnosis, acute appendicitis remains an enigmatic challenge and a remainder of the art of surgical diagnosis.

\section{Materials and methods}

Study design: Case Control Study

Study setting:Tertiary care teaching hospital

period: Cases admitted and treated in General surgery Department In Tertiary care teaching Hospital from May 2016 to November 2017.

Research subjects: All patients admitted with clinical diagnosis of "Acute Appendicitis" or "Appendicular 
perforation" and undergoing surgery in General Surgery Dept in Tertiary care teaching hospital were taken as Subjects for this study. After taking the proposed Informed Consent, data was collected using the questionnaire/ proforma. The primary data for this study were the blood investigations of the patients.

\section{Inclusion criteria}

- All patients undergoing appendicectomy with a clinical diagnosis of acute appendicitis.

- All patients diagnosed appendicectomy with a clinical dignosis of appendicular perforation.

\section{Exclusion criteria}

- All patients documented to have a past history of Jaundice or Liver disease, Chronic alcoholism (i.e. intake of alcohol of $>40 \mathrm{~g} /$ day for Men and $>20 \mathrm{~g} /$ day inWomen for 10 years, Haemolytic disease, Acquired or Congenital biliary disease.

- All patients with positive HBsAg

- All patients with cholelithiasis

- All patients with Cancer of hepato-biliary system

Sample size: Case of acute appendicitis that had undergone appendicectomy from General Surgery wards during the study period fitting the inclusion criteria.

Formula for sample size $=2(\mathrm{PxQ} / \mathrm{D} 2)$

$\mathrm{P}=$ Proportion of perforated appendix with hyperbilirubinemia is

$=1.96$ when error is $5 \%$

$\mathrm{Q}=100-\mathrm{P}$

$\mathrm{D}$ is $20 \%$ of $\mathrm{P}$

Sample Size $=1.96 \times 1.96 \times 50 \times 50 / 10 \times 10=$ approx 100 .

The following tests were carried out for patients diagnosed as 'Acute Appendicitis' or 'Appendicular Perforation' under General Surgery care and admitted to Tertiary care teaching hospital. All blood samples were drawn within 24 hours of admission.

- Routine blood investigations (i.e. complete blood count, platelet count, etc.,)

- Peripheral smear to rule out hemolytic anemia.

- Serum Bilirubin (Total \& Direct bilirubin) $\backslash$

- Liver Enzymes, which include -

- SGPT (Alanine transaminase)

- SGOT (Aspartate transaminase)

- ALP (Alkaline phosphatase)

- Seropositivity for $\mathrm{Hbs} \mathrm{Ag}$

- Urine examination (routine and microscopy)

100 patients with concerned consent and meeting the inclusion criteria were included in the study. The necessary clinical and lab details were obtained in a prewritten format. The intraoperative findings were noted and documented precisely. Upon completion of data collection the preoperative bilirubin levels of the perforated and uncomplicated groups were compared which formed the basis of the study. Other clinical and lab parameters obtained during the study were compared between the two groups. All parameters were subjected to statistical analysis and evaluated critically.

\section{Results}

A total of 100 patients participated in the study. Of the 100 patients studied 66 patients were males and 34 patients were females (Figure - 1). The youngest patient was 13 years old and the oldest 64 years (Table - 1). Among all the patients operated with a diagnosis of Acute Appendicitis 46 were found to have a perforated appendix, and 54 were found to have a non-perforated appendix (Figure-2).

Figure - 1: Male: Female ratio in Appendicitis.

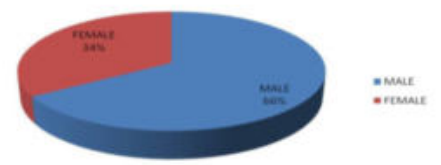

TABLE 1 :Distribution of patients based on Age Interval

\begin{tabular}{|c|c|}
\hline AGE INTERVAL (in yrs) & NO: OF PATIENTS \\
\hline$<21$ & 34 \\
\hline $21-30$ & 39 \\
\hline $31-40$ & 16 \\
\hline $41-50$ & 7 \\
\hline $51-60$ & 2 \\
\hline$>60$ & 2 \\
\hline
\end{tabular}

Figure 2: Acute Appendicitis 46 were found to have a perforated appendix, and 54 were found to have a nonperforated appendix.

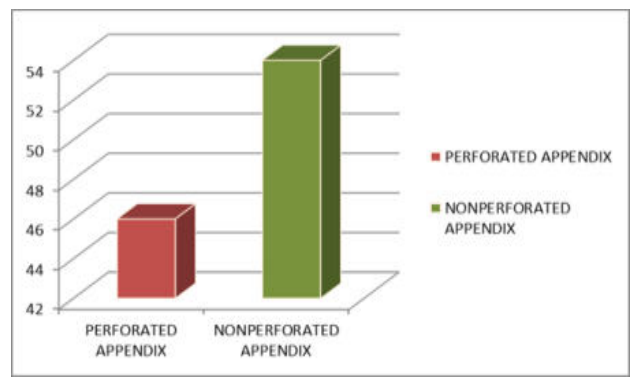

All patients were found to have a raised total leukocyte count and a shift of leukocytes to left. It was observed that among 46 patients with perforated appendix 43 patients showed a raised CRP levels and 29 patients had a raised Bilirubin levels. However in case of non-perforated cases it was found that among the 54 cases 41 patients had a raised CRP levels and 18 patients showed raised bilirubin levels.

Further detailed study revealed that among perforated cases the CRP levels rise observed within the ranges of 1-10, 11-20 and $>20 \mathrm{mg} / \mathrm{dl}$ were in 7,22 and 14 cases respectively. In nonperforated cases the numbers of patients in the biochemical ranges were 19,18 and 9 cases respectively as depicted in Figure-3.

Figure - 3: Proportion of patients with perforated and nonperforated appendix with various intervals of Serum Levels of Total Bilirubin.

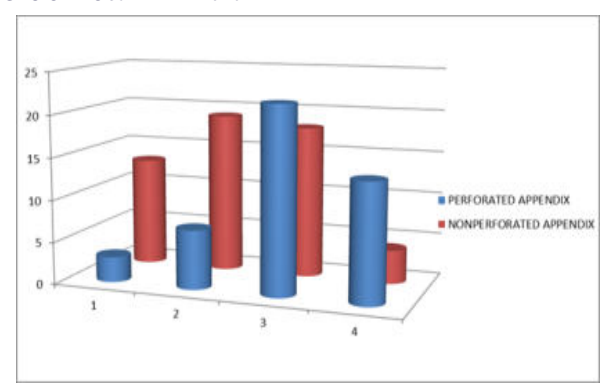

Further analysis revealed that among perforated cases, the Bilirubin levels rise observed within the ranges of 1-2, 2.1-3 and $>3 \mathrm{mg} / \mathrm{dl}$ were in 18,10 and $\mathrm{l}$ cases respectively. In nonperforated cases the number of patients in the biochemical ranges was 13,5 and 0 cases respectively as depicted in Figure-4.

Figure - 4: Proportion of patients with Perforated and Non Perforated Appendix with various intervals of serum levels of CRP. 


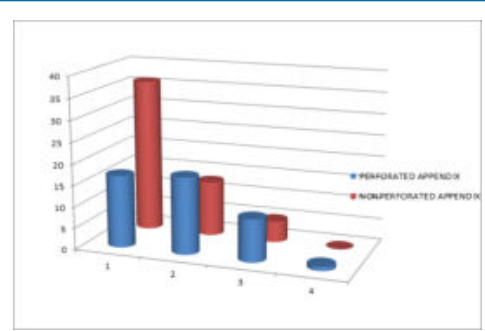

The Comparative analysis by SPSS activity a logistic regression analysis the EXP (B) value were calculated to be 2.647 for Bilirubun and 2.638 for CRP. Hence Logistic Regression technique revealed that the C- reactive protein and Hyperbilirubinemia can be used to predict the appendicular perforation preoperatively and that their roles are comparable and that there is no definitive advantage of one over the other for the same (Table -2).

\section{Table 2:Variables in the Equation}

\begin{tabular}{|c|c|c|c|c|c|c|c|}
\hline & & B & S.E. & Wald & $\mathrm{Df}$ & Sig. & $\operatorname{Exp}(B)$ \\
\hline \multirow[t]{3}{*}{ Step $1^{a}$} & ILIRUBIN & .973 & .453 & 4.623 & 1 & .032 & 2.647 \\
\hline & CRP & .970 & .725 & 1.791 & 1 & .181 & 2.638 \\
\hline & Constant & -1.466 & .641 & 5.241 & 1 & .022 & .231 \\
\hline
\end{tabular}

a. Variable(s) entered on step l: bil, crp.

b. SPSS activity - a logistic regression analysis

\section{Discussion}

Several serum markers have been analysed so as to predict the severity of acute appendicitis including IL- 6 and lipopolysaccharide binding protein [14]. The presence of jaundice in sepsis is well documented, specially associated with gram negative organisms. Several mechanisms have been described explaining elevated total bilirubin levels in systemic infections.

Some bacterias including E.Coli have been associated with increased levels of total serum bilirubin levels [15]. Some endotoxins released in the peripheral blood stream interfere with the liver's mechanism for the bilirubin uptake and canalicular excretion. Endotoxins produce cholestasis by damaging biliary salt transport through cytokine mediated mechanisms. E. Coli is the most frequently isolated bacteria from peritoneal fluid in acute appendicitis. Elevated total bilirubin level in acute appendicitis can either appear as a result of bacteremia, or endotoxemia, both possible in catarrhal and phlegmonous forms as well as in the perforated appendicitis. Several studies had reported elevated levels of serum bilirubin in acute appendicitis. Sand, et al. derived hypothesis that elevated total bilirubin levels can be associated with appendicular perforation [16]. Estrada et al also found out same results [17].

They explain the raised serum bilirubin levels by the invasion of gram negative bacteria through muscularis propria of the appendix, leading to the direct invasion or translocation of germs through portal system and liver, interfering with bilirubin exretion through bile ducts by endotoxin action. 100 patients in whom appendicectomy done were studied.

Several studies have suggested that patients with clinical appendicitis and hyperbilirubinaemia are more likely to have appendiceal perforation. An increased bilirubin level $(\geq 1.5$ $\mathrm{mmol} / \mathrm{L}$ ) was found to have high sensitivity in detecting appendicular perforation. However it did not play any major role as an adjunct to increased TLC and CRP.

TLC and CRP are non-specific inflammatory mediators. A predisease TLC and CRP status of patients in our study was not known, which otherwise would have helped in making a better decision regarding increased reason for increased levels of these mediators. Furthermore, levels of direct and indirect bilirubin were not separately known. Such information would have helped in a more specific analysis.

\section{Conclusions}

Maximum incidence in adults is in the age group 13-30 years. There is a male predominance (2:1 ratio). Tenderness in right iliac fossa is the consistent finding in all the 100 patients. Raised total leukocyte count and shift to left are seen in all cases. The C-reactive protein and Hyperbilirubinemia can be used to predict the appendicular perforation preoperatively and that their roles are comparable and that there is no definitive advantage of one over the other for the same.

\section{References}

1. Flum DR, Morris A, Koepsell T, Dellinger EP. Has misdiagnosis of appendicitis decreased over time? A population based analysis. JAMA, 2001; 286: 748-1753.

2. Andersson RE, Hugander A, Thulin AJ. Diagnostic accuracy and perforation rate in appendicitis: association with age and sex of the patient and with appendicectomy rate. Eur J Surg., 1992; 158:37-41.

3. Vermeulen B, Morabia A, Unger PF. Influence of white cell count on surgical decision making in patients with abdominal pain in the right lower quadrant. Eur JSurg., 1995; 161:483-486.

4. Birchley D. Patients with clinical acute appendicitis should have preoperative full blood count and C-reactive protein assays. Ann R Coll Surg Engl., 2006; 88:27-32.

5. Körner H, Söreide JA, Söndernaa K. Diagnostic accuracy of inflammatory markers in patients operated on for suspected acute appendicitis: a receiver operating characteristic curve analysis. Eur J Surg., 1999;165:679-685.

6. Vaughan-Shaw PG, Rees JR, Bell E, et al. Normal inflammatory markers in appendicitis: evidence from two independent cohort studies. JRSM Short Rep.,2011;2:43.

7. Andersson RE. Meta-analysis of the clinical and laboratory diagnosis of appendicitis. Br J Surg., 2004;91:28-37.

8. Khan S. Elevated serum bilirubin in acute appendicitis: a new diagnostic tool. Kathmandu Univ Med.J,2008;6: 161-165.

9. Estrada JJ, Petrosyan M,Barnhart J, et al. Hyperbilirubinemia in appendicitis: a new predictor of perforation.J Gastrointest Surg., 2007;11:714-718.

10. Sand M, Bechara FG, Holland-Letz T, et al. Diagnostic value of hyperbilirubinemia as a predictive factor for appendiceal perforation in acute appendicitis. Am J Surg., 2009; 198: 193-198.

11. Atahan K, Üreyen O, Aslan E, et al. Preoperative diagnostic role of hyperbilirubinaemia as a marker of appendix perforation. J Int Med Res. 201 1;39:609-618.

12. McGowan DR, Sims HM, Shaikh I, Uheba M. The value of hyperbilirubinaemia in the diagnosis of acute appendicitis. Ann R Coll Surg Engl., 201 1;93:498.

13. Emmanuel A, Murchan P, Wilson I, Balfe P. The value of hy-perbilirubinaemia in the diagnosis of acute appendicitis. Ann R Coll Surg Engl., 2011; 93: 213-217.

14. Branescu C, Serben D, Dascalu A MIL-6 and lipopolysaccharide binding protein markers of inflammation in acute appendicitis. Chirurgia, 2013; 108(2):206-14.

15. Chand N, Sanyal A J. Sepsis induced cholestasis. Hepatology, 2007;45(1):23041 .

16. Sand M, Bechara GF, Holland-Letz T, Sand D, Mehnert G, Mann B. Diagnostic value of Hyperbilirubinemia as a predictive factor for Appendiceal perforation in Acute Appendicitis. Am J Surg., 2009 Aug; 198(2): 193-8.

17. Estrada JJ Petrosyan M, Krumenacker J Jr, Huang S, Moh P. Hyperbilirubinemia in Appendicitis: A New Predicator of Perforation. Journal of Gastrointestinal Surgery, 2007; 11:714-5. 